\title{
La lingua prima, le altre lingue. Quale politica linguistica per la scuola e l'Università?
}

\section{Cristiana De Santis}

\section{(2) OpenEdition \\ 1 Journals}

Edizione digitale

URL: http://journals.openedition.org/esp/4173

DOI: 10.4000/esp.4173

ISSN: 2532-0319

Editore

Centre d'Information sur l'Éducation Bilingue et Plurilingue

\section{Edizione cartacea}

Data di pubblicazione: 1 juin 2019

Paginazione: 91-98

ISSN: 1127-266X

\section{Notizia bibliografica digitale}

Cristiana De Santis, «La lingua prima, le altre lingue. Quale politica linguistica per la scuola e

I'Università?», Éducation et sociétés plurilingues [Online], 46 | 2019, Messo online il 01 février 2021, consultato il 01 mars 2021. URL: http://journals.openedition.org/esp/4173 ; DOI: https://doi.org/ 10.4000/esp.4173

Questo documento è stato generato automaticamente il 1 mars 2021.

(c) CIEBP 


\title{
La lingua prima, le altre lingue. Quale politica linguistica per la scuola e l'Università?
}

\author{
Cristiana De Santis
}

1 Lo scorso 28 novembre si è tenuto a Bologna, presso la Sala Rossa di Palazzo Marchesini, sede del Centro Internazionale di Studi Umanistici "Umberto Eco", un incontro di studio sul tema della politica linguistica e dell'educazione plurilingue che ha visto a confronto Claude Hagège, già Professore del Collège de France, e Francesco Sabatini, già Professore dell'Università di Roma Tre e Presidente Emerito dell'Accademia della Crusca.

2 Claude Hagège, poliglotta e linguista di fama internazionale, è noto come autore di libri che esplorano la struttura, la storia e i destini delle lingue europee, oltre che come pioniere degli studi di tipologia linguistica (con ricerche sul campo effettuate in tutto il mondo). Il suo impegno per la promozione del francese e per la tutela della diversità delle lingue e delle culture è testimoniato da una nutrita serie di volumi (pubblicati in Francia da Odile Jacob, cfr. Hagège nella Bibliografia) che ne fanno uno dei testimoni più autorevoli e incisivi della "lotta" per la sostenibilità linguistica.

3 Francesco Sabatini è noto, oltre che per i suoi studi "fondanti" nel campo della storia della lingua italiana, per il suo impegno nella promozione del multilinguismo nell'ambito dell'Unione Europea. Come Presidente dell'Accademia della Crusca, ha ideato la Settimana della Lingua Italiana nel Mondo, promossa ogni anno dal Ministero degli Affari Esteri italiano, nonché il Progetto "Firenze Piazza delle Lingue d'Europa". A più riprese è intervenuto sul tema del rapporto tra lingue e nazioni in Europa, per valorizzare l'italiano e insieme il "patrimonio linguistico comune degli europei" (dato da una pluralità di lingue di cultura), puntando a un miglioramento generale della comprensione interlinguistica. A questi temi è dedicata la Parte VI (Tomo III) di Sabatini. 2001. Il volume raccoglie 14 scritti dal 1988 al 2008. Sui temi della politica linguistica è tornato inoltre nel suo ultimo libro (Sabatini 2016). 
Oggetto del dibattito a due voci, il delicato equilibrio - a livello nazionale, europeo, mondiale - tra lingue diverse, ciascuna dotata di potere identitario in quanto espressione di una forte tradizione culturale e di una nazione o di una etnia, ma caratterizzate da un diverso valore economico nel cosiddetto "mercato delle lingue". Mercato che oggi vede un'incontestabile egemonia dell'inglese, lingua "invadente" sia per lo spazio che ha conquistato come lingua veicolare negli scambi pratici, sia per lo spazio che tende a erodere in ambito speculativo come lingua internazionale della scienza.

5 Entrambi i relatori hanno sottolineato l'importanza per l'individuo di conoscere più lingue: da un lato, infatti, il monolinguismo mette a rischio il pensiero critico e la creatività intellettuale (Hagège); dall'altro, più lingue si conoscono più si acquisisce quella flessibilità di idee necessaria per spiegare fenomeni complessi, linguistici e non solo (Sabatini); il multilinguismo, inoltre, è uno dei fondamenti della pace perché promuove il desiderio delle nazioni di abbattere le frontiere culturali e politiche (Hagège).

6 Entrambi si sono soffermati sulle implicazioni educative legate alle scelte di politica linguistica, mettendo in luce i rischi connessi con atteggiamenti di snobismo che portano da un lato alla svalutazione dello studio delle lingue prime (con risultati di impoverimento espressivo), dall'altro a una riduzione della gamma di lingue straniere insegnate a scuola, a vantaggio dell'inglese. Entrambi hanno proposto una personale articolazione del percorso scolastico che promuova l'apertura a più varietà di lingue $\mathrm{e} a$ lingue diverse dalla prima (Sabatini) o il bilinguismo precoce (Hagège). Entrambi, infine, hanno rimarcato l'importanza di una formazione adeguata e scientificamente aggiornata degli insegnanti sui temi della linguistica.

7 Nella sua relazione, tenuta in italiano, Hagège si è soffermato sul nesso tra lingua e nazione, portando numerosi esempi a sostegno dell'idea che «l'identificazione di lingua e nazione non è una legge universale»: se è vero, come recita un detto ungherese, che ̀̀ nella sua lingua che vive una nazione, coloro che parlano una certa lingua non per questo sono sempre legati allo Stato di cui essa è espressione ufficiale.

8 Nell'Europa di oggi ci sono nazioni che si sono costituite come tali nonostante le profonde differenze di lingua e cultura degli abitanti (Belgio, Svizzera). Numerose sono inoltre le comunità che, pur restando attaccate alla loro lingua, lo sono ancor di più alla loro identità nazionale (è il caso dei francesi germanofoni di Alsazia, o dei finlandesi che vivono nel sud della Svezia).

9 L'ingresso dei Paesi in Europa è subordinato alla ratifica della dichiarazione dei diritti dell'uomo, che comporta il rispetto delle minoranze e la tutela della diversità linguistica. È tuttavia innegabile che nell'Europa di oggi si assista a un risorgere del nazionalismo, in forme che possono variare: per i paesi più potenti significa espansione e universalismo, per le minoranze si esprime come dissidenza e lotta per affermare la propria identità, anche a livello linguistico (è il caso della Catalogna).

10 Va detto poi che, in alcuni casi, le differenze linguistiche vengono esasperate a causa di vicende storiche precise: è il caso della ex Jugoslavia, in cui la lingua è rimasta più $o$ meno la stessa, nonostante la pressione di croati e serbi per affermare la diversità delle rispettive parlate.

11 È possibile che nel futuro più o meno prossimo si imponga in Europa una lingua comune di tipo federativo adottata dalle istituzioni sovranazionali già esistenti e dagli 
eventuali organi con funzione transeuropea che verranno creati (un esercito comune, per esempio). L'impiego di una lingua comune non significa in alcun modo erosione delle altre lingue né scomparsa delle differenze. La lingua federativa sarà inevitabilmente influenzata dalle lingue materne, che continueranno a dominare nell'uso quotidiano e dovranno essere studiate anche all'estero. L'auspicio è che più europei imparino più lingue possibili.

In quest'ottica, è necessario promuovere un insegnamento multilingue, nella scuola e nell'università. Tre le chiavi proposte a tal fine da Hagège (1996b).

La prima chiave è l'apprendimento precoce di un'altra lingua, a partire dai 6 anni: il momento cioè in cui i bambini sono più duttili, hanno le orecchie più aperte e una curiosità più accesa, perché non ancora imprigionati in quella fortezza costruita dall'insieme dei saperi orientati che definisce la visione degli adulti (a questa opposizione, tra «les blocages de l'adulte et les grâces de l'enfant», è dedicata la prima parte di Hagège 1996b). Questo insegnamento deve basarsi in primo luogo sulla fonetica (prescindendo dalla notazione grafica), senza dimenticare l'intonazione (da insegnare come un gesto articolatorio), e i toni per le lingue che li prevedono ${ }^{1}$.

La seconda chiave proposta è la "strumentalizzazione" della lingua: facendo perno sulla dimensione ludica propria del bambino (cui Hagège allude con la felice espressione puer ludens), bisogna presentare le lingue da imparare non come oggetti artificiali e astratti, ma come strumenti per parlare con coetanei di altri Paesi e per vincere nei giochi (sportivi e non solo; sull'importanza del gioco sia come «moteur d'intégration au groupe» sia come mezzo di costruzione cooperativa del significato si veda Hagège 1996b: 105). Più in generale, si tratta di impiegare le lingue straniere all'interno di relazioni significative, che coinvolgano il corpo e la dimensione dell'affettività.

La terza chiave dovrebbe consistere nella promozione di scambi internazionali di maestri e maestre che consentano di imparare le lingue da parlanti nativi, compensando l'assenza delle lingue studiate nell'ambiente di vita dei bambini. Sarebbe così possibile realizzare nel proprio Paese un'autentica "immersione linguistica" precoce nella lingua straniera, analoga a quella realizzata per il francese nelle scuole valdostane. È necessario che, a monte, gli insegnanti ricevano una formazione in psicopedagogia ma anche in linguistica, sotto forma di iniziazione viva alle caratteristiche tipologiche delle lingue materne degli alunni (che li metta in condizione di capire la ragione degli errori) ${ }^{2}$.

In tema di "immersione linguistica", Hagège si è detto favorevole all'insegnamento disciplinare in una seconda lingua (in Italia conosciuto come CLIL: Content and Language Integrated Learning), nella misura dei $2 / 5$ del programma. La scelta di considerare la lingua straniera moderna come un mezzo di comunicazione e di studio, anziché come una materia insegnata al pari delle altre, consentirebbe di liberare ore da riservare all'insegnamento delle lingue di tradizione classica (latino e greco in Occidente, arabo classico e coranico in Medioriente, cinese classico e confuciano, giapponese classico, sanscrito in oriente). Nei $3 / 5$ delle materie insegnate in lingua materna rientrano sicuramente la lingua e la letteratura nazionale; per quanto riguarda la storia, l'ideale sarebbe un insegnamento bilingue ispirato al principio di complementarità, che al tempo stesso salvaguardi la memoria degli eventi e dei discorsi che hanno forgiato l'identità di un Paese, e arricchisca lo spirito degli alunni presentando visioni differenti dei fatti storici. 

prima, la cui padronanza piena può e deve facilitare un apprendimento più consapevole di altre lingue ${ }^{3}$. Con riferimento alla didattica dell'italiano come lingua materna in Italia, Sabatini lamenta la formazione inadeguata degli insegnanti: debolissima nelle discipline linguistiche e spesso carente anche sul piano delle metodologie didattiche. Ancorare i saperi a presupposti scientifici solidi è il primo presupposto per migliorare gli effetti dell'insegnamento dell'italiano e farne una materia forte. A proposito dell'avvio della riflessione metalinguistica, per esempio, è fondamentale avere consapevolezza della capacità che i bambini hanno (o non hanno ancora) di assimilare un insegnamento esplicito dei meccanismi della lingua. Su questo aspetto insiste anche Hagège nel volume citato: «Une formulation esplicite d'un ensemble de mécanismes constituant un tout logiquement ordonné n'est concevable que si l'enfant, qui est surtout habité du désir de communiquer, est parvenu à une étape intellectuelle qui lui fasse apercevoir l'efficacité d'une connaissance lucide des lois qui soustendent la communication. Or, une telle étape suppose un développement de l'intellection que l'on peut considérer comme déjà proche de la démarche adulte» (Hagège 1996b: 60).

Sabatini ha illustrato quindi un grafico ${ }^{4}$ che sintetizza la sua proposta di educazione linguistica continua riferita a un individuo che vive in un determinato spazio geografico, di cultura e di lingua. Questo spazio, rappresentato come un trapezio con base minore in basso (o, tridimensionalmente, come un tronco di cono) è tagliato in varie sezioni orizzontali che rappresentano i vari ambiti di vita nei quali l'individuo si muove (da quelli più legati alle esigenze primarie si sale verso quelli che comportano una maggiore apertura e proiezione verso l'esterno: i linguaggi non verbali, come la segnaletica e i linguaggi formalizzati). A queste sezioni corrispondono altrettante 
lingue: dalla eventuale lingua locale (in Italia vanno tenute presenti le realtà dialettali, molto vitali, anche se prive di uno standard scritto) si sale verso la lingua nazionale. (La situazione linguistica, ovviamente, può essere più complessa quando lo Stato abbia più lingue nazionali riconosciute, o quando l'individuo appartenga a una minoranza linguistica o parli una lingua di immigrazione). Nella lingua nazionale si sviluppa la consapevolezza metalinguistica, attraverso la riflessione grammaticale, la considerazione storica e sociale della lingua e l'attenzione delle varietà d'uso della lingua in diversi tipi di testi; in tale lingua, inoltre, si compiono le esperienze culturali più significative e si dovrebbe raggiungere una conoscenza profonda della tradizione che in quella lingua si è espressa. Come lingue di "superstrato culturale" sarà possibile introdurre anche lo studio del greco e del latino, finalizzato non solo all'accesso ai testi classici, ma alla comprensione del lessico intellettuale delle lingue moderne.

Naturalmente bisognerà introdurre presto anche una seconda lingua che si presti all'uso internazionale, ed eventualmente aprirsi a una terza lingua, che arricchisca il patrimonio linguistico individuale a prescindere dai bisogni pratici legati alle esigenze della vita moderna, e da considerazioni utilitaristiche legate allo spazio d'uso che una certa lingua abbia conquistato nel mondo.

Se questo è il percorso ipotizzato per un individuo nell'età della formazione, non va dimenticata l'importanza di pianificare una formazione linguistica continua, tenuto conto anche dell'allungamento della vita media: occorrono programmi di formazione linguistica più ricchi e ampi, che vadano ben oltre i primi 20 anni di vita, e che rispondano in modo adeguato ai crescenti bisogni di linguaggio.

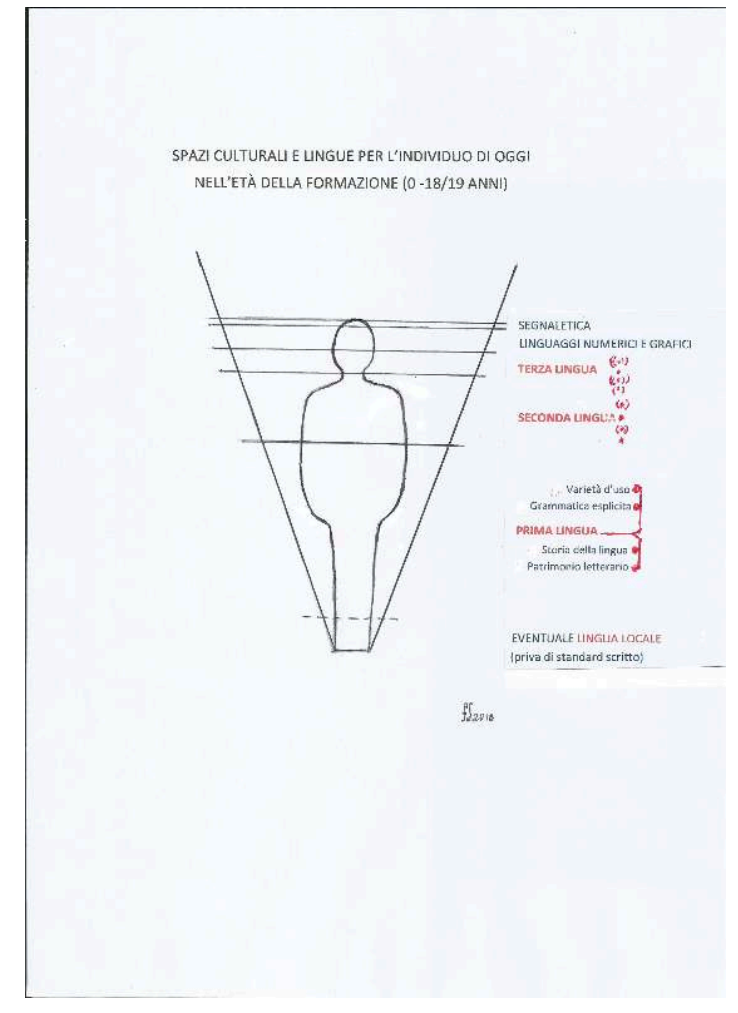




\section{BIBLIOGRAFIA}

HAGÈGE C. 1992. Le Souffle de la langue: voies et destins des parlers d'Europe, Paris, Odile Jacob.

HAGÈGE C. 1996a. Le français, histoire d'un combat, Paris, Odile Jacob.

HAGÈGE C. 1996b. L'Enfant aux deux langues, Paris, Odile Jacob.

HAGÈGE C. 2000. Halte à la mort des langues, Paris, Odile Jacob.

HAGÈGE C. 2006. Combat pour le français: Au nom de la diversité des langues et des cultures, Paris, Odile Jacob.

HAGÈGE C. 2012. Contre la pensée unique, Paris, Odile Jacob.

SABATINI F. 2001. L'italiano nel mondo moderno, Saggi scelti dal 1968 al 2009, Napoli, Liguori.

SABATINI F. 2016. Lezione di italiano. Grammatica, storia, buon uso, Milano, Mondadori.

SABATINI F. 2018, in corso di stampa. Italiano lingua prima, oggi, in Per un'idea di scuola: l'educare, l'insegnare, l'apprendere, Atti del Convegno (Montesilvano, 23-25 marzo 2018), a cura di C. PETRACCA, Teramo, Lisciani.

\section{NOTE}

1. In questa occasione, il linguista non è tornato sulla proposta (formulata in Hagège 1996b) di non includere l'inglese in un sistema di educazione bilingue (limitando le lingue straniere studiate fin dalla scuola primaria a tedesco, spagnolo, francese, italiano, portoghese), e di introdurlo solo nella scuola secondaria come seconda lingua straniera possibile (insieme ad altre grandi lingue non europee). A distanza di vent'anni, l'affermazione incontrastata dell'inglese e le scelte in materia di politica scolastica operate dai governi europei hanno reso difficilmente percorribile questa ipotesi finalizzata alla salvaguardia della diversità linguistica di fronte alla pressione egemonica dell'inglese.

2. Nello stesso volume (Hagège 1996b: 85), il linguista si sofferma sul concetto di «inteferenze linguistiche» derivanti dalla tendenza del bambino a costruire ipotesi interpretative a partire da ciò che gli è più familiare. Si veda anche quanto scrive a proposito della formazione in linguistica richiesta agli insegnanti e dell'esigenza di promuovere atteggiamento flessibile rispetto alla norma della lingua insegnata, che li metta in condizione di accorder aux productions linguistique des enfants una attention exempte de tout dogmatisme » (Hagège 1996b: 126).

3. Anche Hagège ha altrove sottolineato l'importanza delle competenze nella lingua prima: «Le succès de l'immersion n'est pas sans relation avec le niveau de connaissance que les enfants immergés possèdent initialement dans leur propre langue maternelle» (Hagège 1996: 117). La lingua materna fornisce infatti al bambino «les bases cognitives qui fondent son dialogue avec son environnement physique, social, intellectuel» (Hagège 1996b: 118), nonché la consapevolezza metalinguistica necessaria all'apprendimento di altre lingue (Hagège 1996b: 119).

4. Il grafico riprende e sviluppa un analogo prospetto presentato da Sabatini in un saggio del 1988 intitolato titolo Spazi culturali e lingue per l'individuo oggi (apparso in Studi latini e italiani, II, 1988, pp. 183-194 ; ora in Sabatini. 2011. L'italiano nel mondo moderno, Saggi scelti dal 1968 al 2009, Napoli: Liguori, vol. III, pp. 209-219). 


\section{RIASSUNTI}

Cet article offre au lecteur un compte-rendu du séminaire de recherche sur la politique linguistique et de l'éducation plurilingue qui a eu lieu à Bologne le 28 novembre 2018, avec la participation de Claude Hagège (Collège de France) et Francesco Sabatini (Accademia della Crusca). Les orateurs se sont concentrés sur la relation entre la première langue (français ou bien italien) et d'autres langues européennes, y compris une langue "envahissante" telle que l'anglais. Tout en visant à une éducation plurilingue, les deux linguistes ont dessiné une articulation différente du parcours scolaire: pour Hagège il s'agit de promouvoir le bilinguisme précoce et l'immersion linguistique, alors que Sabatini se penche sur une ouverture progressive à plusieurs variétés de la langue maternelle ainsi qu'à différentes langues étrangères. Les deux ont souligné la nécessité d'une formation continue et prolongée dans les langues vivantes étrangères, tout en affirmant l'importance de l'étude des langues classiques. Les deux orateurs ont également insisté sur l'importance de la préparation en linguistique des futurs enseignants.

This article is the full account of the research seminar on language policy and multilingual education held in Bologna on November 28, 2018, with the participation of Claude Hagège (Collège de France) and Francesco Sabatini (Accademia della Crusca). The speakers concentrated on the relationship between the first language (French or Italian, here) and other European languages, including an "intrusive" language such as English. While multilingual education was their focal point, the two linguists sketched a different picture of how it should be done: for Hagège, it means promoting bilingualism and immersion at the earliest possible age, whereas for Sabatini, it should be a progressive exposure to several varieties of the first language as well as of foreign languages. Both stressed the need for a prolonged and continuing education in foreign tongues, as well as the importance of studying the classics. Both also underscored the importance of linguistics in teacher training.

\section{INDICE}

Keywords : language policy, multilingual education, implementation, relation between languages, linguistics

Mots-clés : politique linguistique, éducation plurilingue, organisation, relation entre langues, linguistique

\section{AUTORE \\ CRISTIANA DE SANTIS}

Università de Bologna 